\title{
Performance of Vehicular Visible Light Communications under the Effects of Atmospheric Turbulence with Aperture Averaging
}

\author{
Elizabeth Eso ${ }^{1, *}$, Zabih Ghassemlooy ${ }^{1} \mathbb{D}$, Stanislav Zvanovec ${ }^{2} \mathbb{D}$, Juna Sathian ${ }^{1}$, Mojtaba Mansour Abadi ${ }^{1}$ \\ and Othman Isam Younus ${ }^{1}$ (D)
}

1 Optical Communications Research Group, Faculty of Engineering and Environment, Northumbria University, Newcastle-upon-Tyne NE1 8ST, UK; z.ghassemlooy@northumbria.ac.uk (Z.G.); juna.sathian@northumbria.ac.uk (J.S.); mojtaba.mansour@northumbria.ac.uk (M.M.A.); othman.younus@northumbria.ac.uk (O.I.Y.)

2 Department of Electromagnetic Field, Faculty of Electrical Engineering, Czech Technical University in Prague, 16627 Prague, Czech Republic; xzvanove@fel.cvut.cz

* Correspondence: elizabeth.eso@northumbria.ac.uk

Citation: Eso, E.; Ghassemlooy, Z.; Zvanovec, S.; Sathian, J.; Abadi, M.M.; Younus, O.I. Performance of Vehicular Visible Light Communications under the Effects of Atmospheric Turbulence with Aperture Averaging. Sensors 2021, 21, 2751. https:// doi.org/10.3390/s21082751

Academic Editor: Yang Yue

Received: 9 March 2021

Accepted: 11 April 2021

Published: 13 April 2021

Publisher's Note: MDPI stays neutral with regard to jurisdictional claims in published maps and institutional affiliations.

Copyright: (C) 2021 by the authors. Licensee MDPI, Basel, Switzerland. This article is an open access article distributed under the terms and conditions of the Creative Commons Attribution (CC BY) license (https:// creativecommons.org/licenses/by/ $4.0 /)$.

\begin{abstract}
In this paper, we investigate the performance of a vehicular visible light communications (VVLC) link with a non-collimated and incoherent light source (a light-emitting diode) as the transmitter (Tx), and two different optical receiver (Rx) types (a camera and photodiode (PD)) under atmospheric turbulence (AT) conditions with aperture averaging (AA). First, we present simulation results indicating performance improvements in the signal-to-noise ratio (SNR) under AT with AA with increasing size of the optical concentrator. Experimental investigations demonstrate the potency of AA in mitigating the induced signal fading due to the weak to moderate AT regimes in a VVLC system. The experimental results obtained with AA show that the link's performance was stable in terms of the average SNR and the peak SNR for the PD and camera-based Rx links, respectively with $<1 \mathrm{~dB}$ SNR penalty for both Rxs, as the strength of AT increases compared with the link with no AT.
\end{abstract}

Keywords: vehicular; visible light communication; atmospheric turbulence; aperture averaging; incoherent light source

\section{Introduction}

The currently established radio frequency (RF) technology for vehicular environments known as dedicated short-range communications offers several applications, such as emergency braking and intersection collision warnings [1]. Nevertheless, the RF-based vehicular communications (VC) have a few issues, including (i) lower packet reception rates due to congestion when the number of vehicles on the road is high [1-3]; and (ii) attaining high reliability requirements for intelligent transport systems (ITS) considering the security issues [4]. To address these issues, visible light communications (VLC) technology, as part of optical wireless communication (OWC) technology, has been proposed as a complementary technology to the RF wireless systems in ITS [5]. VLC can be used in a vehicular network environment for vehicle-to-vehicle, vehicle-to-infrastructure, and infrastructure-to-vehicle communications, which are commonly referred to as vehicle-to-everything communications. Moreover, the widespread increase in the use of light-emitting-diode (LED)-based lights in traffic infrastructure, and vehicle headlights and taillights (TLs)-which offer longer life spans, lower dissipation of heat, high-speed switching functionality, and brighter illumination levels than the halogen bulbs used in the past [6] - has made the realization of VLC a possible option in ITS. VLC systems for indoor applications have been intensely investigated over the last decade $[7,8]$; however, their applications in outdoor environments, including VC, are still relatively new and needs further investigations $[9,10]$. In this emerging field of $\mathrm{VC}$, channel modelling is critical to determining the performance boundaries imposed by the models and sizes of vehicles, infrastructure facilities, and real 
outdoor conditions (i.e., fog, atmospheric turbulence (AT), and rain/snow), which will significantly impact the performance of the link and has been sparsely reported in the literature [11]. In this work, the focus is on the AT, which is due to the varying temperature gradients and air pressure. AT is more evident on the road's surface on hot days and around vehicle exhausts.

Among the few studies reported on AT in VLC systems is [12], where the effects of AT on the signal quality by means of simulation considering the signal-to-noise ratio (SNR), bit error rate (BER) performance, and channel capacity metrics of the VLC system were investigated. The results obtained showed that there was a considerable variation in the BER as a function of AT levels; e.g., the BERs at a $100 \mathrm{~m}$ link span for two AT conditions of refractive index structure parameter $C_{n}^{2}$ of $1 \times 10^{-13}$ and $1 \times 10^{-12}$ were $3 \times 10^{-8}$ and $4 \times 10^{-4}$, respectively. In [13], the effects of AT on the BER performance of a VLC system were experimentally investigated under controlled laboratory conditions; however, the strength of AT studied was not characterized or specified. Results obtained showed a slight increment in the BER; e.g., under the same system parameters, the BER rose from $3 \times 10^{-5}$ to $\sim 2 \times 10^{-4}$ for a link with a 128-quadrature amplitude modulation data format. The average BER of the maritime VLC system under oceanic turbulence was evaluated in [14], and it was found that the BER decreased with increasing aperture size and wavelength, and increased with oceanic turbulence and link span. In [15], the effects of fog and weak AT on a VLC link with a camera-based optical receiver $(\mathrm{Rx})$ were investigated using Pearson's correlation coefficient with reference to a template signal. The results obtained showed that under weak AT there was no significant change in the signal quality. In [11], we experimentally investigated the effects of higher AT strengths with AA than in [15], up to $C_{n}^{2}$ of $1.1 \times 10^{-10} \mathrm{~m}^{-2 / 3}$ in vehicular VLC (VVLC) with a camera-based Rx for two camera gain factors (i.e., low and high). Results showed that the peak SNR (PSNR) performance (i.e., an image quality metric) of the link was not significantly degraded by AT with AA for both gain factors investigated.

Note that (i) in VLC systems, depending on applications, two types of Rxs are used: a photodiode (PD) and a camera. (ii) The effects of AT have been reported extensively for OWC links based on a coherent and a highly collimated light source (i.e., lasers) with a $\mathrm{PD}$, as the $\mathrm{Tx}$ and $\mathrm{Rx}$, respectively. There is still the scarcity of information on experimental/simulation studies on the effects of AT in VVLC systems employing incoherent and non-collimated light sources (i.e., LEDs) as the Txs. In this study, we extended our previous works by experimentally investigating the effects of AT with AA on the performance of a VVLC link with a PD-based Rx under weak to moderate AT regimes. Furthermore, we illustrate the effects of beam divergence of the light source on the link performance considering practical VVLC system parameters. It is worth noting that optical wireless systems (visible and infrared bands) employing camera-based Rxs offer relatively lower data rates compared with the high-speed PD-based Rxs, due to the camera frame rates. However, the low data rate $R_{b}$ feature of optical camera communications (OCC) should not be seen as a problem, considering that there are many applications, including VC [16], indoor localization [16], Internet of Things (IoT) [16], sensor networks, motion capturing [17], and advertising [18], where $R_{b}$ is considerably low. In OCC systems, each pixel at the receiving image sensor can detect signals at different wavelengths over the visible range, e.g., red, green, and blue (RGB), hence offering parallel detection capabilities, an adaptive field of view feature, and even enhanced $R_{b}$ using multiple-input multiple-output $[16,19]$ and artificial neural network-based equalizers [20]. In addition, the information carrying light beams from different sources and many directions via the line-of-sight (LOS) [21,22], non-LOS, and/or both paths [23] can be captured using the camera-based Rxs with the extended transmission range [24] compared with the PD-based links.

The remainder of the paper is organized as follows: Section 2 presents the system's description, including AT parameters, models, and the VLC channel. Section 3 presents the simulation results. The experimental setup, signal extraction, and detection are described in Section 4. In Section 5, results are presented. Finally, conclusions are given in Section 6. 


\section{System Description}

\subsection{AT Parameters and Models}

\subsubsection{AT Parameters}

AT is an effect that arises from disparities in both the temperature and pressure of the atmosphere along the communications path. This, therefore, causes variations of the refractive index, which result in both amplitude and phase fluctuations of the propagating light beam, which leads to fading, and consequently reduced SNR and increased BER [25]. $C_{n}^{2}$ (in $\mathrm{m}^{-2 / 3}$ ) is most commonly used to describe the strength of AT [26], which is given by [27]:

$$
C_{n}^{2}=\left(79 \times 10^{-6} \frac{P}{T^{2}}\right)^{2} C_{T}^{2}
$$

where $P$ represents the pressure in millibars, $T$ is the temperature in Kelvin, and $C_{T}^{2}$ is the temperature structure parameter, which is related to the universal 2/3 power law of temperature variations given as [27]:

$$
D_{T}=\left\langle\left(T_{1}-T_{2}\right)^{2}\right\rangle=\left\{\begin{array}{l}
C_{T}^{2} L_{S}^{\frac{2}{3}}, \quad l_{o} \ll L_{T} \ll L_{o} \\
C_{T}^{2} l_{o}^{-\frac{4}{3}} L_{S}^{2}, 0 \ll L_{T} \ll l_{o}
\end{array}\right.
$$

where $L_{T}$ is the separation distance between two points with temperatures of $T_{1}$ and $T_{2}$, and the inner and outer scales of the small temperature variations are denoted by $l_{o}$ and $L_{0}$, respectively.

Another important parameter, which is adopted to reflect the AT regime, is the Rytov variance $\sigma_{R}^{2}$, which indicates the irradiance fluctuations of the optical signal resulting from AT and is given as [27]:

$$
\sigma_{R}^{2}=1.23 C_{n}^{2} k^{\frac{7}{6}} L_{s}^{\frac{11}{6}}
$$

where the wave number $k=\frac{2 \pi}{\lambda}$ ( $\lambda$ is the wavelength), and $L_{S}$ is the link span. The AT conditions are categorized as follows based on $\sigma_{R}^{2}$ [27]:

$$
\begin{array}{ll}
\text { i. } & \text { Weak regime, } \sigma_{R}^{2}<1 ; \\
\text { ii. } & \text { Moderate regime, } \sigma_{R}^{2} \sim 1 ; \\
\text { iii. } & \text { Strong regime, } \sigma_{R}^{2}>1 ; \\
\text { iv. } & \text { Saturation regime, } \sigma_{R}^{2} \rightarrow \infty .
\end{array}
$$

The scintillation index $\sigma_{I}^{2}$ is also used to quantify the normalized intensity variance generated by AT, and it is given by [28]:

$$
\sigma_{I}^{2}=\frac{\left\langle I^{2}\right\rangle}{\langle I\rangle^{2}}-1
$$

where $I$ denotes the intensity of the optical beam at the $\mathrm{Rx}$, and $\langle$.$\rangle represents the ensemble$ average.

\subsubsection{AT Models}

The lognormal AT model is a widely utilized model for the probability density function (PDF) of the randomly fluctuating signal irradiance in weak to moderate AT regimes due to its simplicity. This model, however, underestimates the behavior of the irradiance fluctuations with the increasing AT strength [27]. Consequently, to address the large- and small-scale scintillations in moderate to strong AT conditions, a modified Rytov theory was proposed in [27], where the optical channel was outlined as a function of disturbances arising from small and large-scale atmospheric effects in relation to factors that behave like a modulation process. This model assumes that gamma distributions guide large and small-scale irradiance fluctuations to develop a PDF model of the irradiance in such a way that the parameters used relate to the AT conditions and are still consistent with the 
scintillation theory [27]. The gamma-gamma (GG) model is a suitable model covering the weak to strong AT regime, and its PDF is given by $[29,30]$ :

$$
p(I)=\frac{2(\alpha \beta)^{\frac{\alpha+\beta}{2}}}{\Gamma(\alpha) \Gamma(\beta)} I^{\left(\frac{\alpha+\beta}{2}\right)-1} K_{\alpha-\beta}(2 \sqrt{\alpha \beta I}), I>0
$$

where $\alpha$ and $\beta$ are the effective numbers of large and small-scale eddies of the scattering process, respectively. $\Gamma($.$) and K_{\alpha-\beta}($.$) denote the gamma function and the modified Bessel$ function of the second order $(\alpha-\beta)$, respectively. For the plane wave radiation at the $\mathrm{Rx}, \alpha$ and $\beta$ are given by, respectively [27]:

$$
\begin{aligned}
& \alpha=1 /\left(e^{0.49 \sigma_{I}^{2} / a}-1\right), \\
& \beta=1 /\left(e^{0.49 \sigma_{I}^{2} / b}-1\right)
\end{aligned}
$$

where $a=\left(1+1.11 \sigma_{I}^{\frac{12}{5}}\right)^{\frac{7}{6}}$ and $b=\left(1+0.69 \sigma_{I}^{\frac{12}{5}}\right)^{\frac{5}{6}}$.

Alternatively, a generalized statistical model of the Malaga distribution can be used to model the irradiance fluctuations under all AT conditions [31]. The merits of this model include a closed-form and a mathematically tractable expression for the fundamental channel statistics under all AT regimes, as well as unifying most of the pre-existing statistical models for irradiance fluctuations [31,32]. The PDF of the Malaga distribution is given as [32]:

$$
f I_{\alpha}\left(I_{\alpha}\right)=A \sum_{k^{\prime}=1}^{\beta^{\prime}} a_{k^{\prime}}^{\prime} I_{\alpha}^{\alpha^{\prime}+k^{\prime}}-11 K_{\alpha-k^{\prime}}\left(2 \sqrt{\frac{\alpha^{\prime} \beta^{\prime} I_{\alpha}}{\gamma^{\prime} \beta^{\prime}+\Omega \prime}}\right), I_{\alpha}>0
$$

where

$$
\begin{gathered}
A \triangleq \frac{2 \alpha^{\prime \frac{\alpha^{\prime}}{2}}}{\gamma^{\lambda+\frac{\alpha^{\prime}}{2}} \Gamma\left(\alpha^{\prime}\right)}\left(\frac{\gamma^{\prime} \beta^{\prime}}{\gamma^{\prime} \beta^{\prime}+\Omega \prime}\right)^{\beta^{\prime}+\frac{\alpha^{\prime}}{2}}, \\
a_{k^{\prime}}^{\prime} \triangleq\left(\begin{array}{c}
\beta^{\prime}-1 \\
k^{\prime}-1
\end{array}\right) \frac{\left(\gamma^{\prime} \beta^{\prime}+\Omega \prime\right)^{1-\frac{k^{\prime}}{2}}}{\left(k^{\prime}-1\right) !}\left(\frac{\Omega \prime}{\gamma^{\prime}}\right)^{k^{\prime}-1}\left(\frac{\alpha^{\prime}}{\beta^{\prime}}\right)^{\frac{k^{\prime}}{2}}, \\
\Omega \prime \triangleq \Omega+2 \rho b_{0}+2 \sqrt{2 \rho b_{0} \Omega} \cos \left(\varphi_{A}-\varphi_{B}\right)
\end{gathered}
$$

where $2 b_{0}$ is the average power of the total scatter components, $\beta^{\prime}$ is a natural number representing the amount of AT, $\rho(0<\rho<1)$ is the amount of scattering power that is coupled to the LOS component, $\alpha^{\prime}$ is related to the effective number of large scale cells of the scattering process, $\Omega$ is the average power of the LOS component, $\gamma^{\prime}=2 b_{0}(\rho-1)$, and $\varphi_{A}, \varphi_{B}$ are the LOS phases and coupled to LOS components.

\subsection{VLC System}

For a VLC system, the received signal via the LOS path is given by [33]:

$$
y(t)=\mathcal{R} x(t) \otimes h(t)+n(t),
$$

where $\mathcal{R}$ is the responsivity of the $\mathrm{PD} ; n(t)$ denotes the additive white Gaussian noise, including the sunlight noise, thermal noise, signal, and dark current-related shot noise sources with zero mean and a total variance $\sigma_{T}^{2}$; and $h(t)$ represents the channel impulse response, which is related to the channel DC gain as given by [33]:

$$
H=\int_{-\infty}^{\infty} h(t) \mathrm{d} t
$$


Note that the daytime ambient sunlight-induced shot noise is the dominant noise source in VVLC, which is expressed as [34,35]:

$$
\sigma_{\mathrm{amb}}^{2}=2 q_{e} I_{\mathrm{amb}} B
$$

where $q_{e}$ is the electron charge, and $B$ is the system's bandwidth. Note that for the on-off keying (OOK) data format, $B=R_{b} / 2$ [36]. $I_{\mathrm{amb}}$ is the ambient noise current, and during the daytime $I_{\mathrm{amb}} \approx I_{\text {sun }}$, which can be expressed as [37]:

$$
I_{\text {sun }}=A_{\mathrm{PD}} g(\varphi) \cos (\varphi) \int_{\lambda_{1}}^{\lambda_{n}} P_{\text {sun }}(\lambda) T_{f}(\lambda) \mathcal{R}(\lambda) d \lambda,
$$

where $A_{\mathrm{PD}}$ is the active area the $\mathrm{PD} ; \varphi$ is the incidence angle; $P_{\text {sun }}$ is the solar irradiance; $\lambda_{1}$ and $\lambda_{n}$ are the integration limits, i.e., the wavelength band, which are $405-690 \mathrm{~nm}$ for the visible range; $g$ and $T_{s}$ represent the gain of the optical concentrator (OC) and the transmittance of the optical filter, respectively. Additionally, the thermal noise, signaldependent shot noise at the PD, the PD's dark current noise, and consequently the total noise variances are given, respectively, as [33,36,38]:

$$
\begin{gathered}
\sigma_{\text {therm }}^{2}=\frac{4 k T_{K} B}{R_{L}}, \\
\sigma_{\text {shot }-\mathrm{rs}}^{2}=2 q_{e} \mathcal{R}(\lambda) P_{t} H B \\
\sigma_{\mathrm{dk}}^{2}=2 q_{e} I_{\mathrm{dk}} B, \\
\sigma_{T}^{2}=\sigma_{\text {sun }}^{2}+\sigma_{\text {therm }}^{2}+\sigma_{\text {shot }-\mathrm{rs}}^{2}+\sigma_{\mathrm{dk}}^{2}
\end{gathered}
$$

where $k$ is Boltzmann's constant, $T_{K}$ is the absolute temperature in Kelvin, $R_{L}$ is the load resistance, and $I_{\mathrm{dk}}$ is the dark current. $P_{t}$ denotes the average optical transmit power, and $H$ for the LOS VVLC link with a symmetrical Tx light radiation pattern can be expressed as [33]:

$$
H=\left\{\begin{array}{c}
\frac{(m+1) A_{\mathrm{PD}}}{2 \pi L_{s}^{2}} \cos ^{m}(\theta) T_{S}(\varphi) g(\varphi) \cos (\varphi), \quad 0 \leq \varphi \leq \varnothing \\
0, \quad \varphi>\varnothing
\end{array}\right.
$$

where $\theta$ denotes the irradiance angle, $\varnothing$ is the angular field of view (AFOV) semi-angle of the $\mathrm{Rx}$, and $L_{S}$ is the distance between the Tx and Rx. The $g$ of a non-imaging OC can be expressed as $A_{\text {coll }} / A_{\mathrm{PD}}$ [39], where $A_{\text {coll }}\left(A_{\text {coll }}=\pi D^{2} / 4\right)$ is the collection area of the non-imaging OC, $D$ is the diameter of the $\mathrm{OC}$, and $m$ represents Lambertian order of emission of the Tx, which is given by [33]:

$$
m=-\frac{\ln 2}{\ln \left(\cos \theta_{1 / 2}\right)},
$$

where $\theta_{1 / 2}$ is the half power angle. Lambertian radiant intensity is expressed as [33]:

$$
R(\phi)=\frac{(m+1)}{2 \pi} \cos ^{m}(\theta),
$$

The average SNR without and with the AT for a point Rx (i.e., with no OC) for OOK signaling are given, respectively, as [27,33]:

$$
\begin{gathered}
\operatorname{SNR}(0)_{0}=\frac{\left(P_{t} \mathcal{R} H(0)\right)^{2}}{\sigma_{T}^{2}(0)}, \\
\operatorname{SNR}(0)_{\mathrm{AT}}=\frac{\mathrm{SNR}_{0}}{\sqrt{1+\sigma_{I}^{2}(0) \mathrm{SNR}_{0}^{2}}},
\end{gathered}
$$


Using an OC (i.e., aperture averaging), the average SNR with and without the AT are given as $[27,33]$ :

$$
\begin{gathered}
\operatorname{SNR}(D)_{0}=\frac{\left(P_{t} \mathcal{R} H(D)\right)^{2}}{\sigma_{T}^{2}(D)}, \\
\operatorname{SNR}(D)_{\mathrm{AT}}=\frac{\operatorname{SNR}(D)_{0}}{\sqrt{1+\sigma_{I}^{2}(D) \operatorname{SNR}(D)_{0}^{2}}},
\end{gathered}
$$

$\sigma_{I}^{2}(D)$ is the variance of the intensity fluctuations for an OC of $D$, and $\sigma_{I}^{2}(0)$ is the scintillation index for a point $\operatorname{Rx}(D \approx 0)$. For the plane wave propagation with the outerand inner-scale temperature variation parameters of $\approx \infty$ and $\approx 0$, respectively, $\sigma_{I}^{2}(D)$ can be expressed as [40]:

$$
\sigma_{I}^{2}(D)=\exp \left[\frac{0.49 \sigma_{R}^{2}}{\left(1+0.653 d^{2}+1.11 \sigma_{R}^{\frac{12}{5}}\right)^{\frac{7}{6}}}+\frac{0.51 \sigma_{R}^{2}\left(1+0.69 \sigma_{R}^{\frac{12}{5}}\right)^{-\frac{5}{6}}}{1+0.9 d^{2}+0.621 d^{2} \sigma_{R}^{\frac{12}{5}}}\right]-1,
$$

where $d=\sqrt{\frac{k D^{2}}{4 L_{s}}}$.

The average BER is given as [33]:

$$
\mathrm{BER}=Q(\sqrt{\mathrm{SNR}})
$$

where $Q(x)$ is the $Q$-function used for the calculation of the tail probability of the standard Gaussian distribution given by [33]:

$$
Q(x)=\frac{1}{\sqrt{2 \pi}} \int_{x}^{\infty} e^{\frac{-y^{2}}{2}} d y,
$$

Next, with (23), (24), and (28) the average BER of the link under AT can be expressed as [27,33]:

$$
\mathrm{BER}_{\mathrm{AT}}=Q\left(\frac{P_{t} \mathcal{R} H}{\sqrt[4]{\left(\sigma_{T}^{2}\right)^{2}+\sigma_{I}^{2}(D)\left(P_{t} \mathcal{R} H\right)^{4}}}\right)
$$

In OCC systems, an image quality metric known as the PSNR is used for assessing the link's quality, which is given by [41]:

$$
\mathrm{PSNR}=10 \log \frac{I_{\mathrm{mx}}^{2}}{\mathrm{MSE}}
$$

where $I_{\mathrm{mx}}=255$ for the grayscale image (i.e., the maximum possible pixel value), and MSE is the pixel luminance mean squared error, which is defined by [16]:

$$
\operatorname{MSE}=\frac{1}{n} \sum_{1=\mathrm{j}}^{n}\left(I_{\mathrm{Tx}}(j)-I_{\mathrm{Rx}}(j)\right)^{2},
$$

where $I_{\mathrm{Tx}}$ are the pixel values for the transmitted symbols, (in this work we have obtained $I_{\mathrm{Tx}}$ from captured images at approximately a zero distance from the Tx, i.e., without considering the channel impact), $I_{R x}$ are the average pixel values for the received symbols, $n$ is the number of rows (i.e., the on and off states of the Tx for OOK signaling scheme), and $j$ is the pixel's row index number.

\section{Simulation of AT Effects with AA}

To reduce the degradation of the propagating signal due to AT effects, a number of options have been proposed, including (i) spatial diversity with adequate spacing [42], 
which is not suitable in VVLC since the width of vehicles is within the range 1.5-2.0 m [43]; (ii) beam width optimization [44], where the radiation pattern of the beam is altered, which is also impractical in VVLC since vehicles will have different HL and TL shapes, dimensions, and radiation patterns; in addition, including additional optics in HL and TL or in another infrastructure lighting to change the radiation properties may not be a viable option; (iii) complex modulation and coding [45]; and (iv) AA technique [40], which is a simple method and can be easily achieved in a VVLC system on the Rx side. AA involves using a lens in front of a small optical detector, thereby increasing the collection area of the $\mathrm{Rx}$, hence lowering the effects of AT and spatially filtering the high fluctuations of the received optical beam. The AA factor is expressed as [40] $\frac{\sigma_{I}^{2}(D)}{\sigma_{I}^{2}(0)}$.

We present here simulation results for the degree of light collimation (i.e., the beam divergence angle $\theta_{1 / 2}$ ) of a light source with AA and under weak to moderate turbulence conditions using Equations (14)-(30). Using the key system parameters given in Table 1, first, we investigate the dependency of the mean BER performance on $\theta_{1 / 2}$ of the Tx without and with weak to moderate AT (i.e., $\sigma_{R}^{2}=0.5$ ) for a range of inter-vehicle distances with $D$ of $25 \mathrm{~mm}$ under sunlight, as depicted in Figure 1. The plot shows that the BER increased with the inter-vehicle distance $L_{S}$ crossing the forward error correction (FEC) limit of $3.8 \times 10^{-3}$ at $L_{s}$ of 50 and $64 \mathrm{~m}$ for $\theta_{1 / 2}$ of $50^{\circ}$, with and without AT, respectively. Moreover, for the $L_{S}$ of $40 \mathrm{~m}$, the BERs without and with AT are $4.3 \times 10^{-4}$ and $3.1 \times 10^{-3}$ for $\theta_{1 / 2}=60^{\circ}$; and $<1.0 \times 10^{-6}$ and $1.0 \times 10^{-3}$ for $\theta_{1 / 2}=30^{\circ}$, respectively.

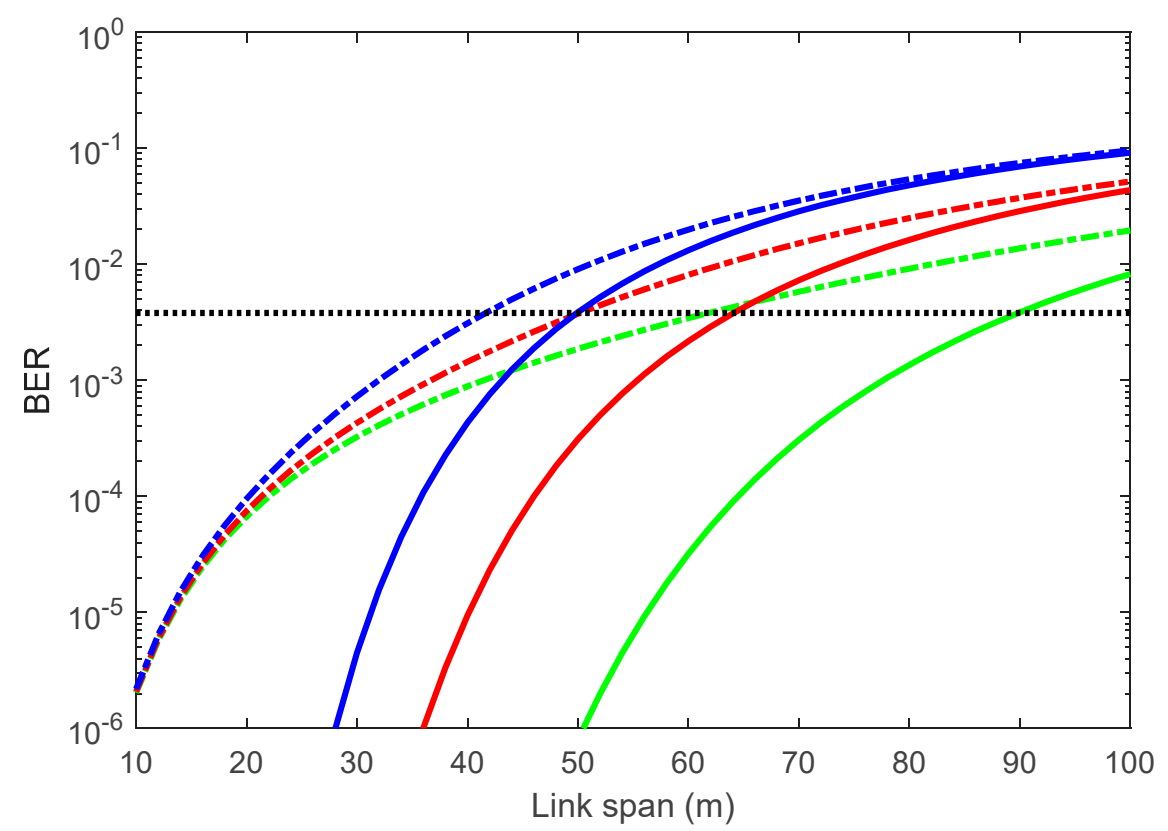

Figure 1. Bit error rate (BER) performance without and with weak to moderate atmospheric turbulence (AT) conditions for a range of inter-vehicle distances and $\theta_{1 / 2}$.

Furthermore, we investigate the average SNR as a function of $\theta_{1 / 2}$ for a range of $D$ under weak to moderate AT (i.e., $\sigma_{R}^{2}=0.5$ ) and $L_{S}$ of $50 \mathrm{~m}$, as shown in Figure $2 \mathrm{a}$. Additionally, shown for reference is the SNR plot for the case with no AT and OC. As shown, the SNR plots are almost independent of $\theta_{1 / 2}$ with AA but increases with $D$ under AT. E.g., for $D=30 \mathrm{~mm}$ and $\theta_{1 / 2}=20,30$, and $40^{\circ}$, the SNRs were 23.9, 23.8, and $23.4 \mathrm{~dB}$, respectively. Next, the average SNR performance gain achieved by AA under AT can be expressed as:

$$
G_{\mathrm{SNR}-\mathrm{AA}}=\frac{\operatorname{SNR}_{\mathrm{AT}}(D)}{\operatorname{SNR}_{\mathrm{AT}}(0)}=\frac{\sqrt{1+\sigma_{I}^{2}(0) \operatorname{SNR}(0)_{0}^{2}}\left(\operatorname{SNR}_{0}(D)\right)}{\sqrt{1+\sigma_{I}^{2}(D) \operatorname{SNR}(D)_{0}^{2}}\left(\operatorname{SNR}_{0}(0)\right)}
$$


where $\mathrm{SNR}_{\mathrm{AT}}(D)$ and $\mathrm{SNR}_{\mathrm{AT}}(0)$ are the mean SNRs with and without AA under AT. Figure $2 \mathrm{~b}$ shows $G_{\mathrm{SNR}-\mathrm{AA}}$ as a function of $D$ for a range of $\sigma_{R}^{2}$, where $L_{s}=50 \mathrm{~m}$ and $\theta_{1 / 2}=45^{\circ}$. It is apparent that $G_{\mathrm{SNR}-\mathrm{AA}}$ increases with a decrease in $\sigma_{R}^{2}$ (AT strength) and an increase in $D$. For example, at $\sigma_{R}^{2}=0.4$, the $G_{\mathrm{SNR}-\mathrm{AA}} \mathrm{s}$ were $14.3,17.5,19.9$, and $21.8 \mathrm{~dB}$ for $D$ of 20,25, 30, and $35 \mathrm{~mm}$, respectively.

Table 1. Key simulation parameters.

\begin{tabular}{cc}
\hline Parameter & Value \\
\hline PD responsivity $\mathcal{R}$ & $0.43 \mathrm{~A} / \mathrm{W}$ \\
\hline Rytov variance $\sigma_{R}^{2}$ & $0.1-1$ \\
\hline Beam divergence angle (half power angle) $\theta_{1 / 2}$ & $30-60^{\circ}$ \\
\hline Inter - vehicle distance $L_{s}$ & $10-100 \mathrm{~m}$ \\
\hline Bandwidth $B$ & $5 \mathrm{MHz}$ \\
\hline Optical transmit power $P_{t}$ & $0.5 \mathrm{~W}$ \\
\hline Diameter of OC $D$ & $20-35 \mathrm{~mm}$ \\
\hline Tx wavelength $\lambda$ & $630 \mathrm{~nm}$ \\
\hline PD area $A_{\mathrm{PD}}$ & $0.75 \times 10^{-4} \mathrm{~m}^{2}$ \\
\hline Diameter of PD & $9.8 \times 10^{-3} \mathrm{~m}$ \\
\hline Incidence & $0^{\circ}$ \\
\hline Absolute temperature $T_{K}$ & $298 \mathrm{~K}$ \\
\hline Solar irradiance $P_{\text {sun }}$ & $100 \mathrm{~W} / \mathrm{m}^{2}$ \\
\hline Load resistance $R_{L}$ & $50 \mathrm{ohms}$ \\
\hline PD dark current $I_{\mathrm{dk}}$ & $5 \mathrm{nA}$ \\
\hline Transmission coefficient of filter $T_{S}$ & 1 \\
\hline
\end{tabular}

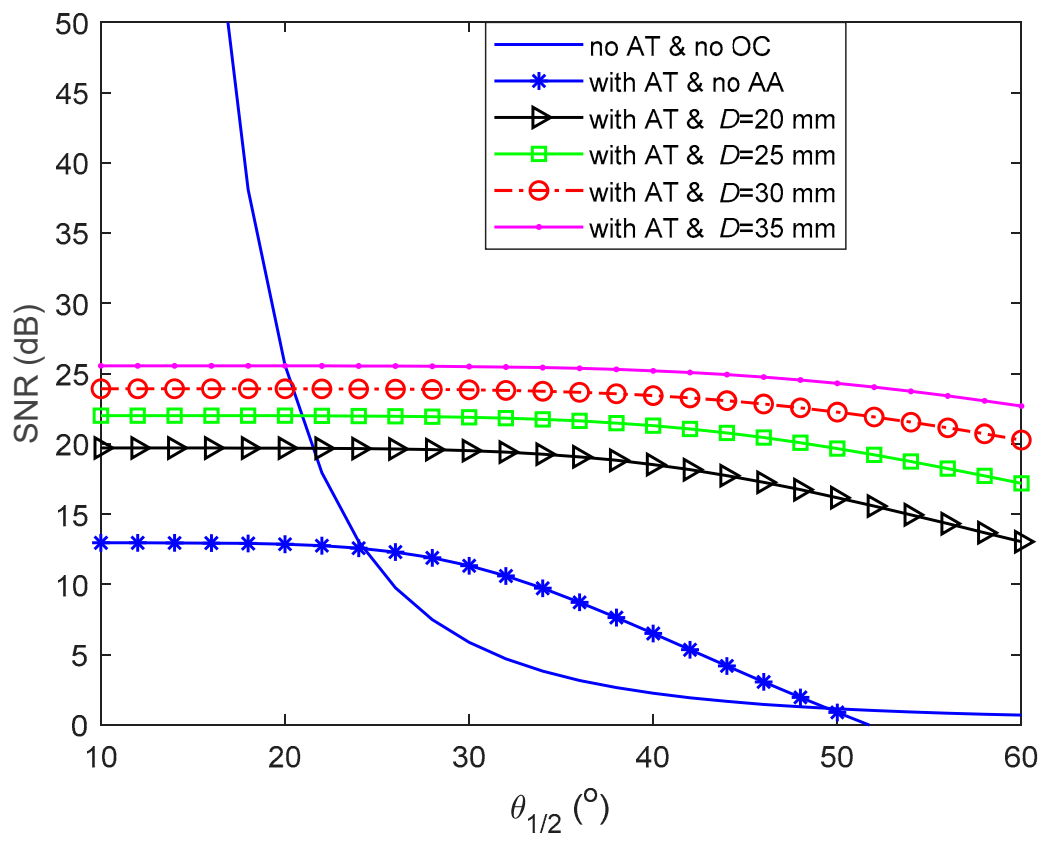

(a)

Figure 2. Cont. 


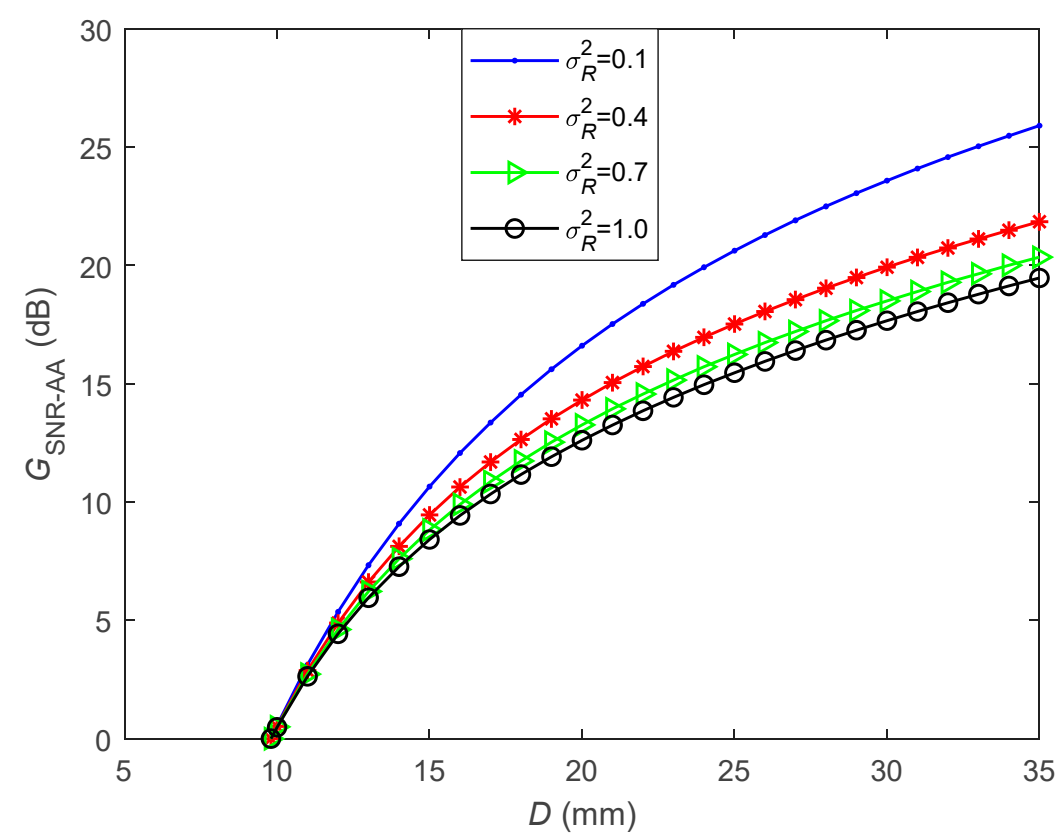

(b)

Figure 2. (a) Effects of aperture averaging (AA) for a range of $D$ on the signal to noise ratio (SNR) performance as a function of $\theta_{1 / 2}$, and (b) the SNR gain as a function of $D$ with AA for a range of $\sigma_{R}^{2}$.

\section{System Setup}

\subsection{Experimental Testbed}

Experimental investigation of AT with AA was carried out. The schematic block diagram of the proposed VVLC system with the PD and camera-based Rxs with AA and under AT is depicted in Figure 3. Rx1 was composed of a camera (Thorlabs DCC1645C-HQ) with a lens (Computar MLH-10X), and Rx2 was composed of a PD (PDA100A2) and a convex lens. An indoor laboratory atmospheric chamber was used to simulate the outdoor $\mathrm{AT}$, as proposed in [26]. At the Tx, data packets of length 90 bits in the non-return to zero OOK format were generated using an arbitrary waveform generator, the output of which was used for intensity modulation of the TL (Truck-trailer DACA08712AM) via the driver module. The intensity-modulated light beam transmitted over a dedicated atmospheric chamber was captured at the Rxs. AT was generated within the chamber by varying the temperature along the transmission path using hot/cold fans. Seventeen temperature sensors were used within the chamber to measure the temperature distribution. The key experimental parameters adopted in the proposed system are listed in Table 2.

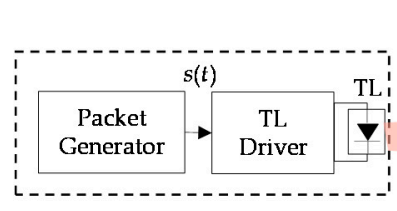

$\mathrm{Tx}$

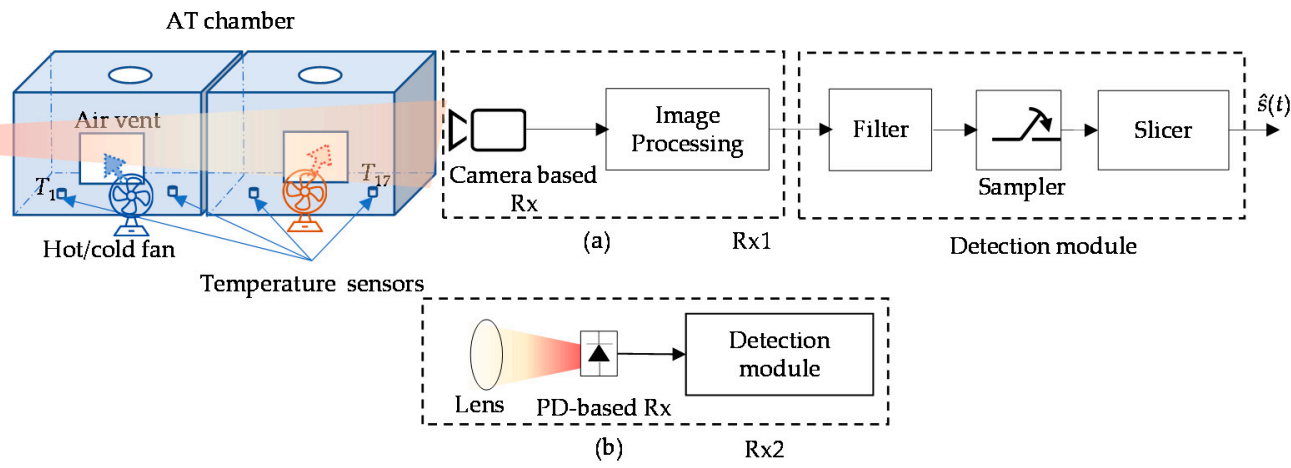

Figure 3. Setup of experimental investigation on AT effects on the vehicular visible light communications (VVLC) link with: (a) the camera and (b) the photodiode (PD) optical receiver (Rx). 
Table 2. Key parameters of the experiment.

\begin{tabular}{|c|c|c|}
\hline \multicolumn{2}{|c|}{ Description } & Value \\
\hline \multirow{3}{*}{$\mathrm{Tx}$} & Tx peak wavelength & $630 \mathrm{~nm}$ \\
\hline & Tx bias current & $98 \mathrm{~mA}$ \\
\hline & Transmit power & $32.4 \mathrm{~mW}$ \\
\hline \multirow{7}{*}{ PD Rx } & \multicolumn{2}{|c|}{ Thorlabs PDA100A2 } \\
\hline & Responsivity & $0.43 \mathrm{~A} / \mathrm{W}$ at $630 \mathrm{~nm}$ \\
\hline & PD area & $0.75 \times 10^{-4} \mathrm{~m}^{2}$ \\
\hline & Bandwidth@ 0 dB gain & $11 \mathrm{MHz}$ \\
\hline & Noise equivalent power @ 960 nm & $7.17 \times 10^{-11} \mathrm{~W} \sqrt{ } \mathrm{Hz}$ \\
\hline & Lens focal length $f$ & $25 \mathrm{~mm}$ \\
\hline & Lens diameter $D$ & $25 \mathrm{~mm}$ \\
\hline \multirow{9}{*}{ Camera Rx } & \multicolumn{2}{|c|}{ Thorlabs DCC1645C-HQ } \\
\hline & Camera shutter speed & $600 \mu \mathrm{s}$ \\
\hline & Camera gain factors & $1.07 \times, 3.96 \times$ \\
\hline & Lens focal length $f$ & $130 \mathrm{~mm}$ \\
\hline & Lens aperture & $f 5.6$ \\
\hline & Samples per frame & 2588 \\
\hline & Pixel clock & $10 \mathrm{MHz}$ \\
\hline & Camera frame rate & $6.25 \mathrm{fps}$ \\
\hline & Camera resolution & $1280 \times 1024$ \\
\hline \multirow{3}{*}{ Packet Generator } & Data format & NRZ-OOK \\
\hline & Packet generator sample rate & $11.125 \mathrm{kHz}$ \\
\hline & Number of samples per bit $n_{\text {sam }}$ & 10 \\
\hline Channel & AT chamber dimension & $33 \times 35 \times 720 \mathrm{~cm}^{3}$ \\
\hline
\end{tabular}

\subsection{Signal Extraction}

On the Rx side, the captured regenerated electrical signals from PD and camera-based Rxs were processed offline in MATLAB ${ }^{\circledR}$. Algorithm 1 shows the image processing algorithm for signal extraction for the camera Rx. For Rx1, a rolling shutter (RS)-based camera was employed; the captured frames' RGB components were converted to grayscale for both the data and calibration video streams following pixelation (i.e., digitizing the image to obtain the pixel value) using Algorithm 1. Note that the data and calibration video streams represent the captured transmitted data and the template shape of the Tx (i.e., the DC gain), respectively. The latter was used for the intensity compensation (i.e., normalization) of the data video frames. Subsequently, the captured frames were then normalized over the rows to obtain the received signal waveform as illustrated in Figure $4 a, b$, which shows an example of a captured frame before and after normalization, respectively. Note that in RS-based cameras, the camera sequentially integrates incoming light illuminating the camera pixels, thereby offering flicker-free transmission with increased data rates. 

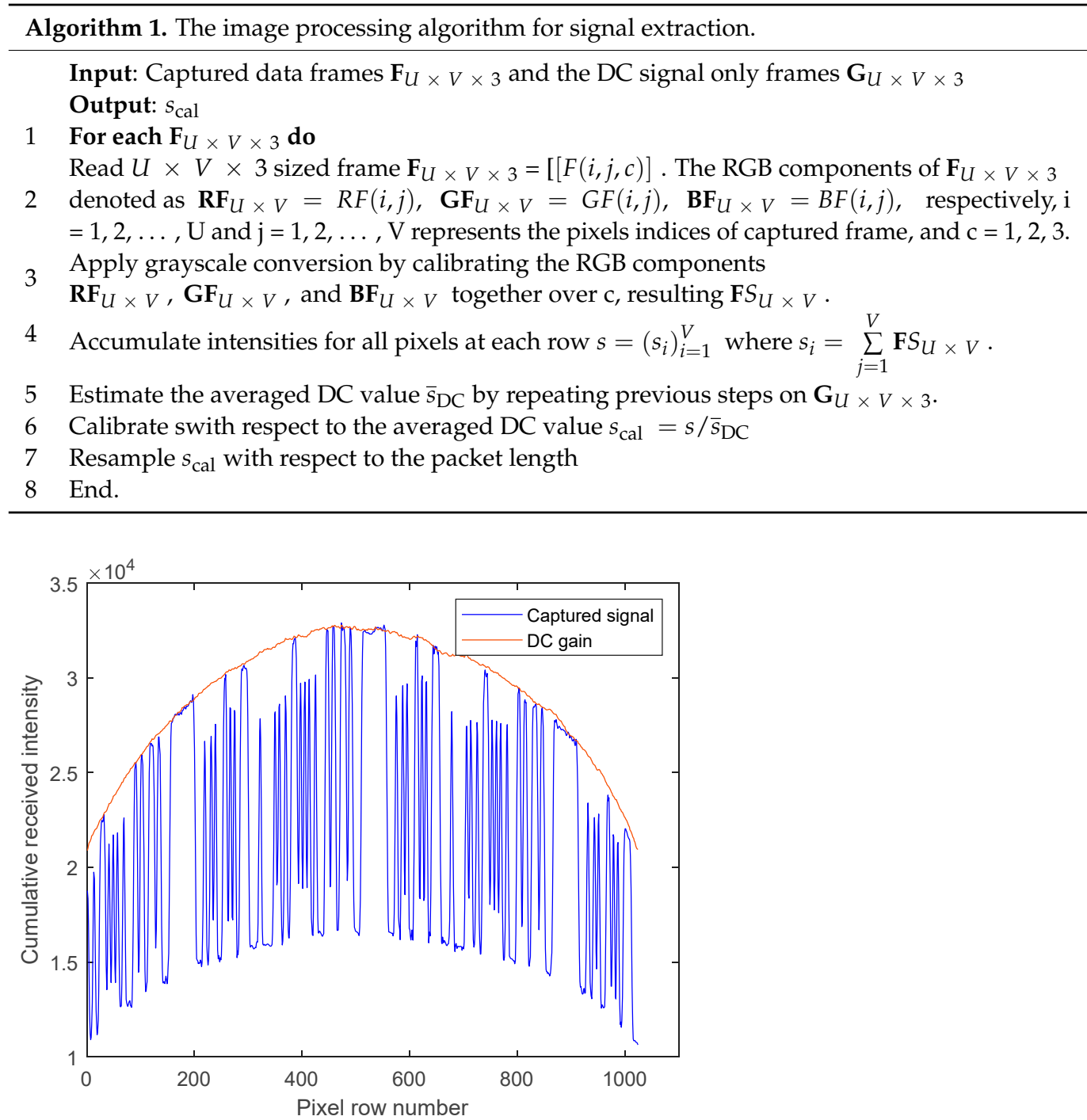

(a)

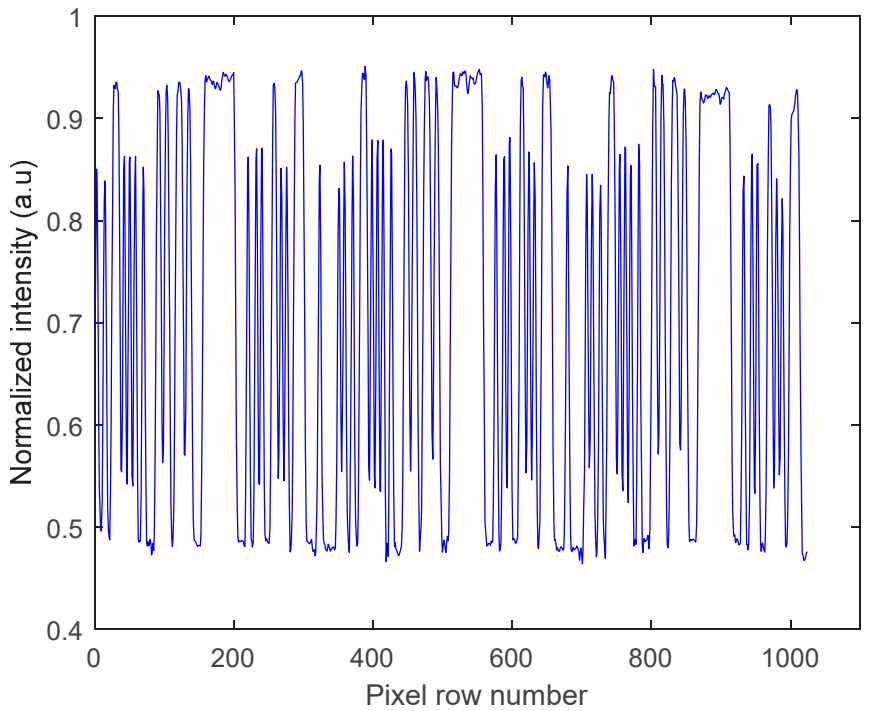

(b)

Figure 4. An example of a captured image frame for the camera-based Rx: (a) before and (b) after intensity normalization. 
For Rx2, i.e., the PD-based Rx, the signal was captured using a digital oscilloscope (Agilent Technologies DSO9254A) for offline processing in MATLAB ${ }^{\circledR}$. For both Rxs, the detection process included (i) a low pass Butterworth filter (first-order with a normalized cut off frequency $w_{n}$ of $0.1 \pi \mathrm{rad} /$ sample for the PD received signals, and a third-order with $w_{n}=0.25 \pi \mathrm{rad} /$ sample for the camera signals are used); (ii) a sampler (sampling at the middle of the samples received for each bit and at an interval of $n_{\text {sam }}$ ); and (iii) a slicer (with the threshold level set to the mean value of the signal, and for Rx1 this was done per frame).

\section{Results and Discussions}

\subsection{Camera-Based $R x$}

Measurements were carried out for link with and without AT for the camera shutter speed of $600 \mu \mathrm{s}$ and the low and high gain factors of 1.07 times $(\times)$ and $3.96 \times$, respectively. Note that the camera-based $\mathrm{Rx}$ had a gain factor in the range of $1 \times$ to $4.27 \times$; where $1 \times$ and $4.27 \times$ imply no gain and the maximum gain, respectively. Consequently, to assess the link quality, we have used the PSNR as given in Equations (31) and (32). Figure 5 shows the PSNR versus $C_{n}^{2}$ with AA for the low and high gain factors. It illustrates that with AA (i) the PSNR is almost independent of AT with a marginal drop for the link with a gain factor of 3.96 $\times$; and (ii) there was a PSNR gain of $\sim 7 \mathrm{~dB}$ for the captured frames at the higher gain factor.

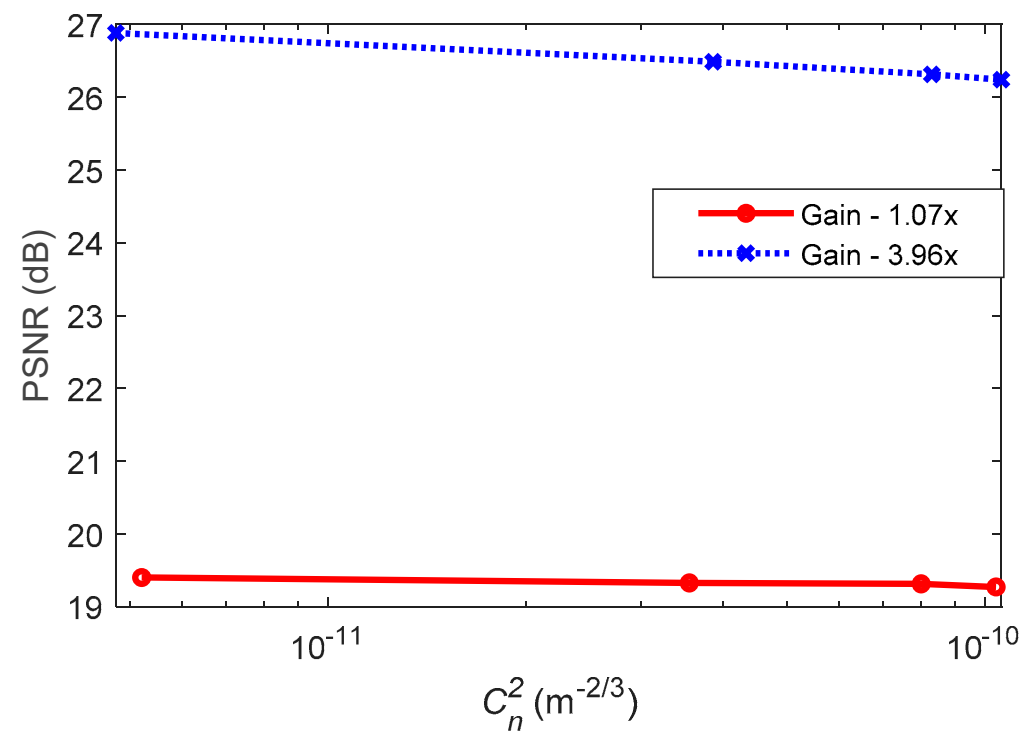

Figure 5. The peak SNR (PSNR) as a function of $C_{n}^{2}$ for the two gain factors.

Note that the corresponding $\sigma_{R}^{2}$ for the experimentally measured $C_{n}^{2}$ for different AT strengths were determined using Equation (3) for $L_{S}$ equal to the length of the AT chamber $(7.2 \mathrm{~m})$, which are given in Table 3 . Note that both the image contrast and its brightness increase with the camera's gain factor, since the signal is amplified prior to the digitizing process [46]. The choice of the two gain factors (i.e., low and high) is used to illustrate the image contrast/brightness effects under AT.

Figure 6 shows the captured received signal waveforms prior to being applied to the detection module for different AT strengths with AA after normalization. The received signal employing a higher gain factor showed improved PSNR performance and higher received signal intensity with lower intensity fluctuations. The BER measured was less than the target BER of $10^{-4}$ for all the link scenarios considered. Furthermore, the histogram plots prior to the detection module are depicted in Figure $7 \mathrm{a}-\mathrm{d}$, which illustrate the distributions of discrete intensity levels of the captured images within the range of 0 to 1 for the captured image frames per link. Figure $7 \mathrm{e}$ shows the histogram for the sampled received intensity 
levels of Figure 7a at the output of the sampler (i.e., within the detection module). Note the slight overlap between the received intensities for the bits 0 and 1 in Figure $7 a-d$, which was due to the slow rise-time of the captured on and off states of the Tx. This was because of the transition between different illumination levels brought about by the sampling process in the RS-based camera [20]. Consequently, this happened at the transition edges; however, for the proposed detection module, the sampling takes place at the center of the received samples per bit; hence the system's performance was not degraded and bits 0 are 1 were clearly distinguishable, as in Figure 7e. Moreover, it can be observed that the link with a higher gain factor (i.e., Figure 7c,d) had higher peak counts than Figure 7a,b with the low gain factor.
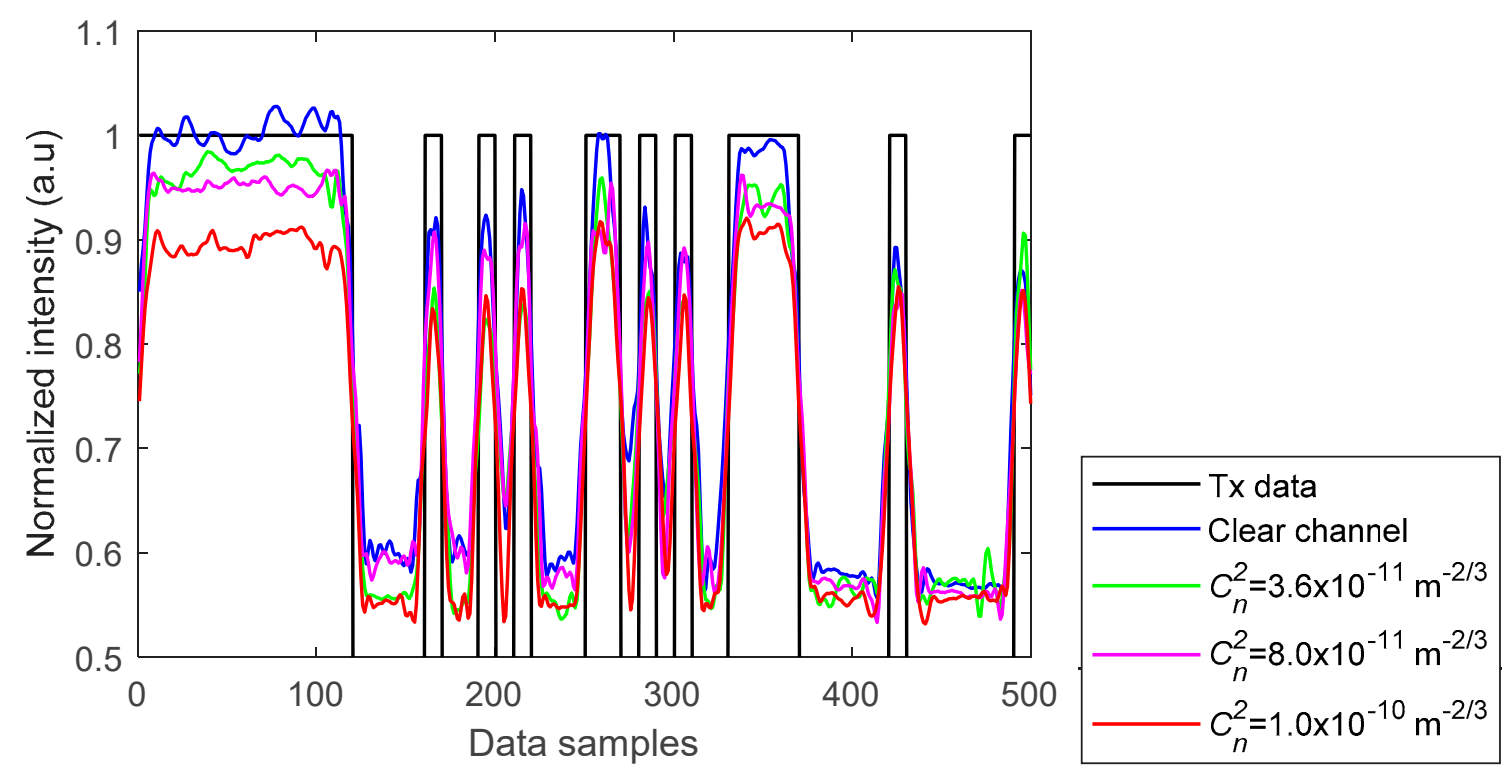

(a)

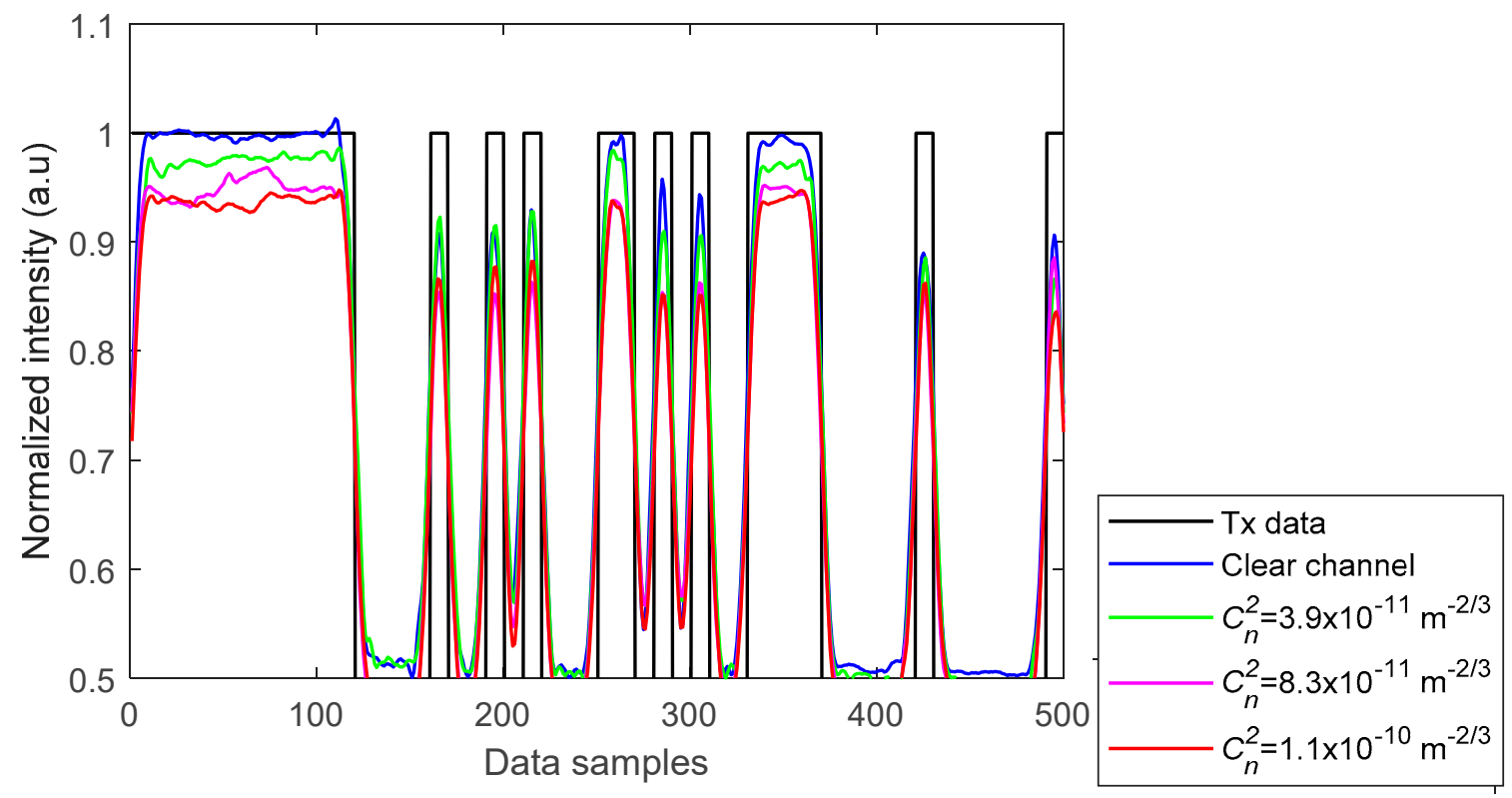

(b)

Figure 6. Waveforms of Rx data at varying AT strengths for the gain factors of: (a) $1.07 \times$ and (b) $3.96 \times$ [11]. 
Table 3. $\sigma_{R}^{2}$ for corresponding $C_{n}^{2}$ values measured in the experiments.

\begin{tabular}{ccc}
\hline & $C_{n}^{2}\left(m^{-2 / 3}\right)$ & $\sigma_{R}^{2}$ \\
\hline \multirow{3}{*}{ PD } & $3.9 \times 10^{-11}$ & 0.2619 \\
\cline { 2 - 3 } & $7.9 \times 10^{-11}$ & 0.5304 \\
\cline { 2 - 3 } & $1.0 \times 10^{-10}$ & 0.6714 \\
\cline { 2 - 3 } & $3.6 \times 10^{-11}$ & 0.2417 \\
\cline { 2 - 3 } & $3.9 \times 10^{-11}$ & 0.2619 \\
\cline { 2 - 3 } & $7.9 \times 10^{-11}$ & 0.5304 \\
\cline { 2 - 3 } & $8.0 \times 10^{-11}$ & 0.5371 \\
\cline { 2 - 3 } & $8.3 \times 10^{-11}$ & 0.5573 \\
\hline
\end{tabular}

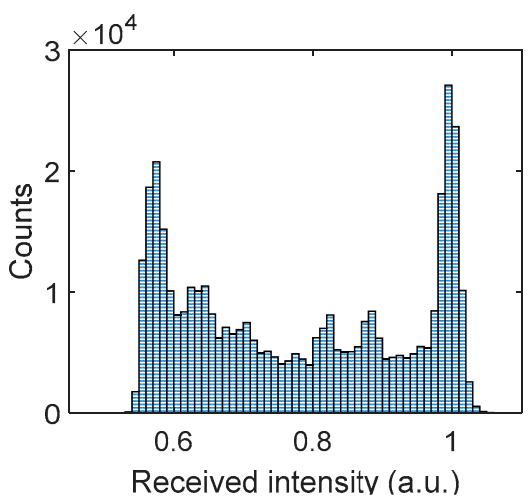

(a)

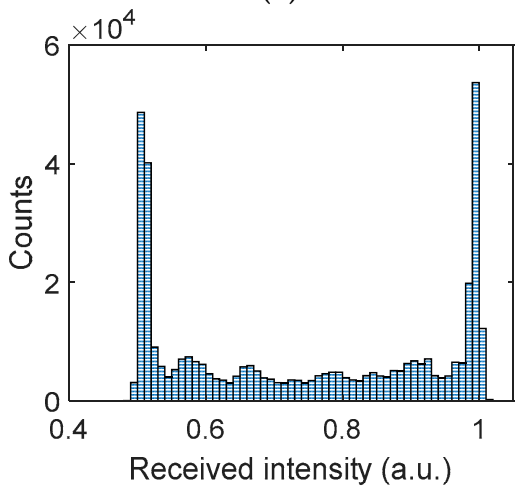

(c)

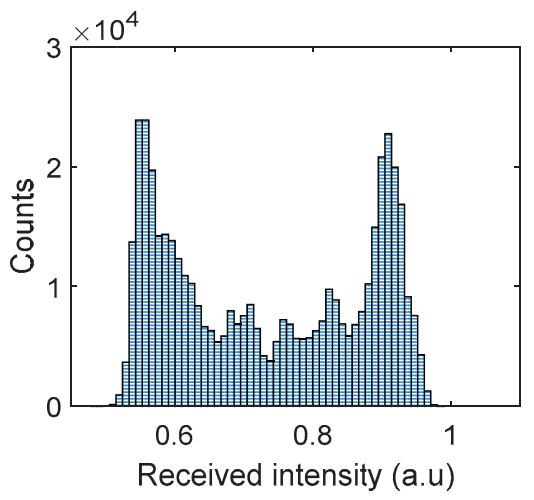

(b)

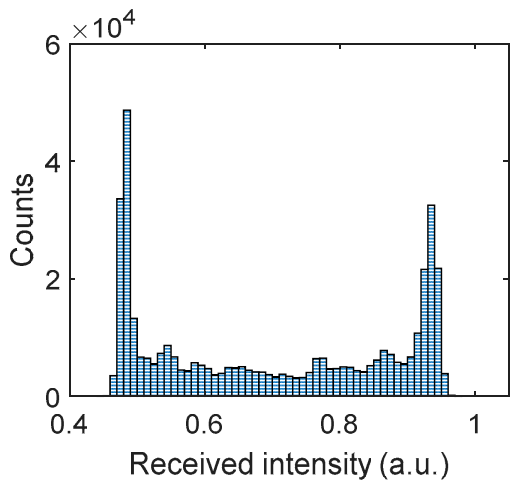

(d)

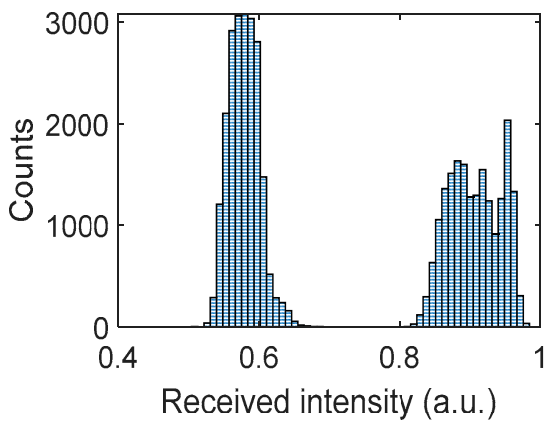

(e)

Figure 7. Camera Rx histogram plot: prior detection module for (a) gain $1.07 \times$ with no AT, (b) gain $1.07 \times$ with the highest AT scenario, $C_{n}^{2}=1.0 \times 10^{-10} \mathrm{~m}^{-2 / 3},(\mathbf{c})$ gain $3.96 \times$ with no AT, and (d) gain $3.96 \times$, with the highest AT scenario $C_{n}^{2}=1.1 \times 10^{-10} \mathrm{~m}^{-2 / 3}$; and (e) output of the sampler for (a). 


\subsection{PD-Based Rx}

For the link with AA and the PD-based Rx, we have measured the SNR of the captured signals and produced histogram plots for bits 0 and 1, i.e., the signal distribution profile for the channel with and without AT, as shown in Figure 8, prior to the detection module. From the results obtained, (i) the histogram plot shows a clear distinction between the received signal for bits 0 and 1 ; and the average SNR is independent of the weak to moderate AT (with only $\sim 0.1 \mathrm{~dB}$ of SNR penalty compared with the clear channel with OC). Thus, this demonstrates that AA can effectively combat the induced signal fading due to AT for the VVLC systems under weak to moderate turbulence regimes.

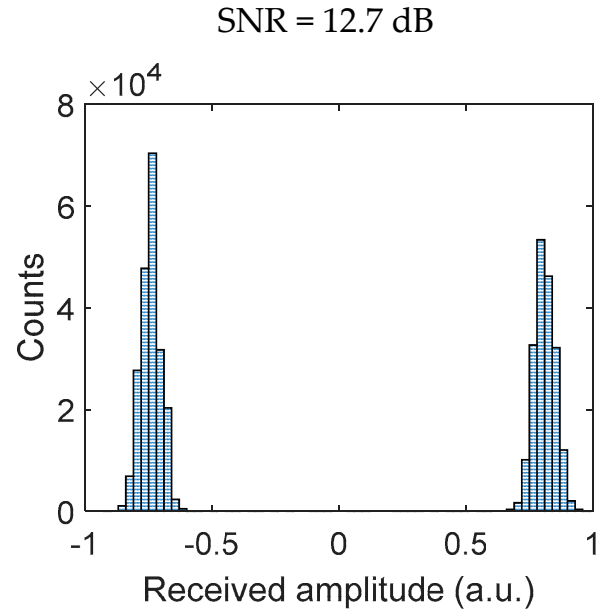

(a)

$\mathrm{SNR}=12.6 \mathrm{~dB}$

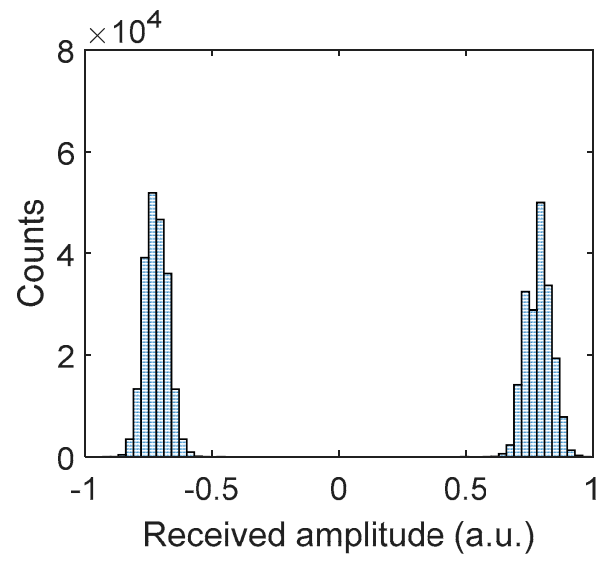

(c)

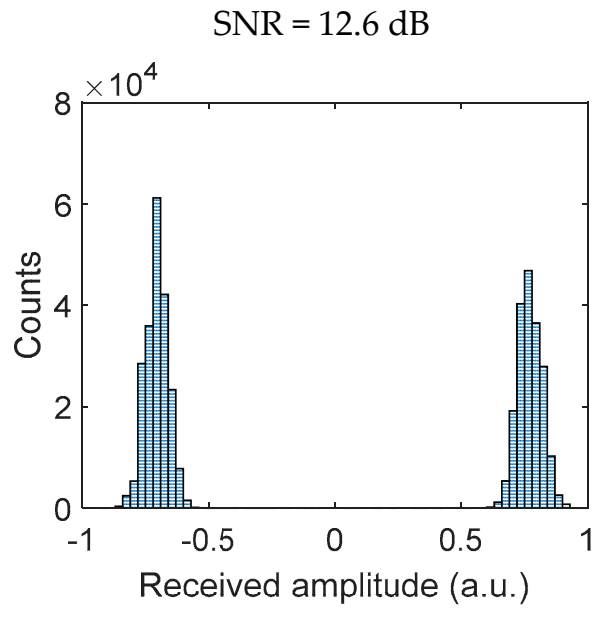

(b)

$\mathrm{SNR}=12.6 \mathrm{~dB}$

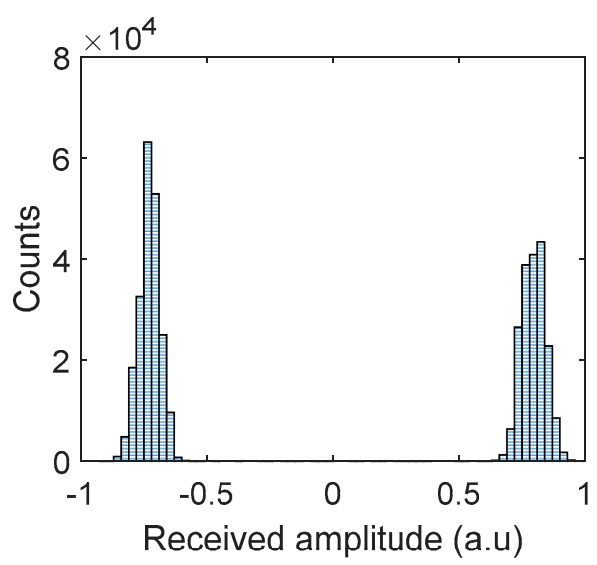

(d)

Figure 8. PD Rx histogram plot for (a) a clear channel and channels with AT conditions of $(\mathbf{b}) C_{n}^{2}=$ $3.9 \times 10^{-11} \mathrm{~m}^{-2 / 3}$, (c) $C_{n}^{2}=7.9 \times 10^{-11} \mathrm{~m}^{-2 / 3}$, and (d) $C_{n}^{2}=1.0 \times 10^{-10} \mathrm{~m}^{-2 / 3}$.

\section{Conclusions}

First, we presented the simulation results for a VVLC system with aperture averaging to mitigate the signal degradation due to atmospheric turbulence. The results obtained showed a performance improvement in terms of the SNR under weak to moderate turbulence regimes with an increasing diameter of the receiver lens. Moreover, results also revealed that, for an increasing beam divergence angle (half power angle) the inter-link BER degradation decreased with and without turbulence. Furthermore, we experimentally investigated the effects of aperture averaging for the VVLC link under turbulence using an LED-based vehicle's taillight. The results obtained showed that with the aperture averaging 
there was no significant system performance degradation under atmospheric turbulence, whereas both PSNR and SNR dropped by 0.7 and $0.1 \mathrm{~dB}$ for the camera and PD- Rxs, respectively, compared with the clear channel. Finally, we demonstrated that in VVLC systems employing incoherent non-collimated LED light sources as the Tx, the aperture averaging method proved to be very potent at mitigating weak to moderate turbulence regimes, and in fact also increased the optical power density of the received signal at the Rx.

Author Contributions: Conceptualization, E.E.; methodology, E.E.; software, E.E. and M.M.A.; validation, E.E., Z.G., M.M.A., and S.Z., investigation, E.E.; resources, Z.G. and M.M.A.; writingoriginal draft preparation, E.E.; writing—review and editing, E.E., Z.G., S.Z., J.S., O.I.Y., and M.M.A.; supervision, Z.G.; funding acquisition, Z.G. All authors have read and agreed to the published version of the manuscript.

Funding: The work was supported by the European Union's Horizon 2020 Research and Innovation Programme under the Marie Sklodowska-Curie grant agreement number 764461 (VISION) and the EU COST Action on Newfocus (CA19111), and partly by CELSA project PICNIC.

Institutional Review Board Statement: Not applicable.

Informed Consent Statement: Not applicable.

Data Availability Statement: The data presented in this study are available on request from the corresponding author.

Conflicts of Interest: The authors declare no conflict of interest. The funders had no role in the design of the study; in the collection, analyses, or interpretation of data; in the writing of the manuscript, or in the decision to publish the results.

\section{References}

1. Gao, S.; Lim, A.; Bevly, D. An empirical study of DSRC V2V performance in truck platooning scenarios. Digit. Commun. Netw. 2016, 2, 233-244. [CrossRef]

2. Shen, W.-H.; Tsai, H.-M. Testing vehicle-to-vehicle visible light communications in real-world driving scenarios. In Proceedings of the 2017 IEEE Vehicular Networking Conference (VNC), Torino, Italy, 27-29 November 2017; pp. 187-194.

3. Vivek, N.; Srikanth, S.V.; Saurabh, P.; Vamsi, T.P.; Raju, K. On field performance analysis of IEEE 802.11p and WAVE protocol stack for V2V \& V2I communication. In Proceedings of the International Conference on Information Communication and Embedded Systems (ICICES2014), Chennai, India, 27-28 February 2014; pp. 1-6.

4. Xu, Z.; Li, X.; Zhao, X.; Zhang, M.H.; Wang, Z. DSRC versus 4G-LTE for Connected Vehicle Applications: A Study on Field Experiments of Vehicular Communication Performance. J. Adv. Transp. 2017, 2017, 1-10. [CrossRef]

5. Bazzi, A.; Masini, B.M.; Zanella, A.; Calisti, A. Visible light communications as a complementary technology for the internet of vehicles. Comput. Commun. 2016, 93, 39-51. [CrossRef]

6. Car Lighting District. Halogen vs. HID vs. LED—Which is Best? 2018. Available online: https:/ / www.carlightingdistrict.com/ blogs/news/halogen-vs-hid-vs-led-which-is-best (accessed on 1 April 2019).

7. Karunatilaka, D.; Zafar, F.; Kalavally, V.; Parthiban, R. LED Based Indoor Visible Light Communications: State of the Art. IEEE Commun. Surv. Tutor. 2015, 17, 1649-1678. [CrossRef]

8. Pathak, P.H.; Feng, X.; Hu, P.; Mohapatra, P. Visible Light Communication, Networking, and Sensing: A Survey, Potential and Challenges. IEEE Commun. Surv. Tutor. 2015, 17, 2047-2077. [CrossRef]

9. Cailean, A.M.; Dimian, M. Impact of IEEE 802.15. 7 Standard on Visible Light Communications Usage In Automotive Applications. IEEE Commun. Mag. 2017, 55, 169-175. [CrossRef]

10. Cailean, A.-M.; Dimian, M. Current Challenges for Visible Light Communications Usage in Vehicle Applications: A Survey. IEEE Commun. Surv. Tutor. 2017, 19, 2681-2703. [CrossRef]

11. Eso, E.; Younus, O.I.; Ghassemlooy, Z.; Zvanovec, S.; Abadi, M.M. Performances of Optical Camera-based Vehicular Communications under Turbulence Conditions. In Proceedings of the 2020 12th International Symposium on Communication Systems, Networks and Digital Signal Processing (CSNDSP), Porto, Portugal, 20-22 July 2020; pp. 1-5.

12. Guo, L.-D.; Cheng, M.-J. Visible light propagation characteristics under turbulent atmosphere and its impact on communication performance of traffic system. In Proceedings of the 14th National Conference on Laser Technology and Optoelectronics (LTO 2019), Shanghai, China, 17 May 2019; 2019; Volume 11170, p. 1117047.

13. Martinek, R.; Danys, L.; Jaros, R. Visible Light Communication System Based on Software Defined Radio: Performance Study of Intelligent Transportation and Indoor Applications. Electron. 2019, 8, 433. [CrossRef] 
14. Zheng, X.-T.; Guo, L.-X.; Cheng, M.-J.; Li, J.-T. Average BER of Maritime Visible Light Communication System in Atmospheric Turbulent Channel *. In Proceedings of the 2018 Cross Strait Quad-Regional Radio Science and Wireless Technology Conference (CSQRWC), Xuzhou, China, 21-24 July 2018; pp. 1-3. [CrossRef]

15. Matus, V.; Eso, E.; Teli, S.R.; Perez-Jimenez, R.; Zvanovec, S. Experimentally Derived Feasibility of Optical Camera Communications under Turbulence and Fog Conditions. Sensors 2020, 20, 757. [CrossRef]

16. Cahyadi, W.A.; Chung, Y.H.; Ghassemlooy, Z.; Hassan, N.B. Optical Camera Communications: Principles, Modulations, Potential and Challenges. Electronics 2020, 9, 1339. [CrossRef]

17. Teli, S.R.; Zvanovec, S.; Ghassemlooy, Z. Performance evaluation of neural network assisted motion detection schemes implemented within indoor optical camera based communications. Opt. Express 2019, 27, 24082-24092. [CrossRef]

18. Saeed, N.; Guo, S.; Park, K.-H.; Al-Naffouri, T.Y.; Alouini, M.-S. Optical camera communications: Survey, use cases, challenges, and future trends. Phys. Commun. 2019, 37, 100900. [CrossRef]

19. Teli, S.R.; Matus, V.; Zvanovec, S.; Perez-Jimenez, R.; Vitek, S.; Ghassemlooy, Z. Optical Camera Communications for IoT-RollingShutter Based MIMO Scheme with Grouped LED Array Transmitter. Sensors 2020, 20, 3361. [CrossRef]

20. Younus., O.I.; Hassan, N.B.; Ghassemlooy, Z.; Haigh, P.A.; Zvanovec, S.; Alves, L.N.; Le Minh, H. Data Rate Enhancement in Optical Camera Communications Using an Artificial Neural Network Equaliser. IEEE Access 2020, 8, 42656-42665. [CrossRef]

21. Lee, H.-Y.; Lin, H.-M.; Wei, Y.-L.; Wu, H.-I.; Tsai, H.-M.; Lin, K.C.-J. RollingLight: Enabling Line-of-Sight Light-to-Camera Communications. In Proceedings of the 13th Annual International Conference on Mobile Systems, Applications, and Services, Florence, Italy, 19-22 May 2015; pp. 167-180.

22. Nguyen, T.; Islam, A.; Hossan, T.; Jang, Y.M. Current Status and Performance Analysis of Optical Camera Communication Technologies for 5G Networks. IEEE Access 2017, 5, 4574-4594. [CrossRef]

23. Hassan, N.B.; Ghassemlooy, Z.; Zvanovec, S.; Biagi, M.; Vegni, A.M.; Zhang, M.; Luo, P. Non-Line-of-Sight MIMO Space-Time Division Multiplexing Visible Light Optical Camera Communications. J. Lightw. Technol. 2019, 37, 2409-2417. [CrossRef]

24. Eso, E.; Teli, S.; Hassan, N.B.; Vitek, S.; Ghassemlooy, Z.; Zvanovec, S. 400 m rolling-shutter-based optical camera communications link. Opt. Lett. 2020, 45, 1059-1062. [CrossRef]

25. Ghassemlooy, Z.; Popoola, W.; Rajbhandari, S. Optical Wireless Communication: System and Channel Modelling with MATLAB, 2nd ed.; CRC Press: Boca Raton, FL, USA, 2019.

26. Nor, N.A.M.; Fabiyi, E.; Abadi, M.M.; Tang, X.; Ghassemlooy, Z.; Burton, A.; Mohd, N.N.A. Investigation of moderate-to-strong turbulence effects on free space optics A laboratory demonstration. In Proceedings of the 201513 th International Conference on Telecommunications (ConTEL), Graz, Austria, 13-15 July 2015; pp. 1-5.

27. Andrews, L.C.; Phillips, R.L. Laser Beam Propagation through Random Media, 2nd ed.; SPIE Press: Washington, DC, USA, 2005.

28. Ghassemlooy, Z.; Le Minh, H.; Rajbhandari, S.; Perez, J.; Ijaz, M. Performance Analysis of Ethernet/Fast-Ethernet Free Space Optical Communications in a Controlled Weak Turbulence Condition. J. Light. Technol. 2012, 30, 2188-2194. [CrossRef]

29. Andrews, L.; Phillips, R.; Hopen, C. Theory of Optical Scintillation with Applications, 1st ed.; SPIE Optical Engineering Press: Bellingham, WA, USA, 2001.

30. Al-Habash, M.; Phillips, R.; Andrews, L. Mathematical model for the irradiance probability density function of a laser beam propagating through turbulent media. Opt. Eng. 2001, 40, 1554-1562. [CrossRef]

31. Sandalidis, H.G.; Chatzidiamantis, N.D.; Karagiannidis, G.K. A Tractable Model for Turbulence- and Misalignment-Induced Fading in Optical Wireless Systems. IEEE Commun. Lett. 2016, 20, 1904-1907. [CrossRef]

32. Jurado-Navas, A.; Garrido-Balsells, J.M.; Paris, J.F.; Castillo-Vazquez, M.; Puerta-Notario, A. Further insights on Málaga distribution for atmospheric optical communications. In Proceedings of the 2012 International Workshop on Optical Wireless Communications (IWOW), Pisa, Italy, 22 October 2012; pp. 1-3.

33. Kahn, J.M.; Barry, J.R. Wireless infrared communications. Proc. IEEE 1997, 85, 265-298. [CrossRef]

34. Komine, T.; Nakagawa, M. Fundamental analysis for visible-light communication system using LED lights. IEEE Trans. Consum. Electron. 2004, 50, 100-107. [CrossRef]

35. Hui, R. Introduction to Fiber-Optic Communications; Academic Press: Cambridge, MA, USA, 2020; pp. 125-154.

36. Haddad, O.; Khalighi, A.; Zvanovec, S. Channel Characterization for Optical Extra-WBAN Links Considering Local and Global User Mobility; SPIE OPTO: San Francisco, CA, USA, 2020.

37. Farahneh, H.; Kamruzzaman, S.M.; Fernando, X. Differential Receiver as a Denoising Scheme to Improve the Performance of V2V-VLC Systems. In Proceedings of the 2018 IEEE International Conference on Communications Workshops (ICC Workshops), Kansas City, MO, USA, 20-24 May 2018; pp. 1-6.

38. Paudel, R. Modelling and Analysis of Free Space Optical Link for Ground-to-Train Communications. Ph.D. Thesis, Northumbria University, Newcastle upon Tyne, UK, 2014.

39. O'Brien, D.C.; Faulkner, G.; Le Minh, H.; Bouchet, O.; El Tabach, M.; Wolf, M.; Walewski, J.W.; Randel, S.; Nerreter, S.; Franke, M.; et al. Gigabit optical wireless for a Home Access Network. In Proceedings of the 2009 IEEE 20th International Symposium on Personal, Indoor and Mobile Radio Communications, Tokyo, Japan, 13-16 September 2009; pp. 1-5.

40. Khalighi, M.-A.; Schwartz, N.; Aitamer, N.; Bourennane, S. Fading Reduction by Aperture Averaging and Spatial Diversity in Optical Wireless Systems. J. Opt. Commun. Netw. 2009, 1, 580-593. [CrossRef]

41. Huynh-Thu, Q.; Ghanbari, M. Scope of validity of PSNR in image/video quality assessment. Electron. Lett. 2008, 44, 800-801. [CrossRef] 
42. Yang, G.; Khalighi, M.-A.; Ghassemlooy, Z.; Bourennane, S. Performance evaluation of receive-diversity free-space optical communications over correlated Gamma-Gamma fading channels. Appl. Opt. 2013, 52, 5903-5911. [CrossRef]

43. Nationwide Vehicle Contracts. Understanding Car Size and Dimensions. 2020. Available online: www.nationwidevehiclecontracts. co.uk/blog/understanding-car-size-and-dimensions (accessed on 1 March 2021).

44. Lee, I.E.; Ghassemlooy, Z.; Ng, W.P.; Khalighi, M.-A.; Liaw, S.-K. Effects of aperture averaging and beam width on a partially coherent Gaussian beam over free-space optical links with turbulence and pointing errors. Appl. Opt. 2015, 55, 1-9. [CrossRef]

45. Uysal, M.; Li, J.; Yu, M. Error rate performance analysis of coded free-space optical links over gamma-gamma atmospheric turbulence channels. IEEE Trans. Wirel. Commun. 2006, 5, 1229-1233. [CrossRef]

46. IDS Imaging and Development Systems GmbH: Brighter Images Thanks to gain- How to work with gain. Available online: https:/ / en.ids-imaging.com/tl_files/downloads/techtip/TechTip_Gain_EN.pdf (accessed on 13 January 2020). 